\title{
Efficacy of dual-functional liposomes containing paclitaxel for treatment of lung cancer
}

\author{
RONG-HUA WANG ${ }^{1}$, HONG-MEI CAO ${ }^{2}$, ZHI-JU TIAN ${ }^{2}$, BO JIN ${ }^{1}$, QING WANG ${ }^{3}$, HONG MA ${ }^{4}$ and JING WU $^{5}$
}

\author{
${ }^{1}$ Department of Cardiothoracic Surgery, ${ }^{2}$ Pharmacy Intravenous Admixture Services and \\ Departments of ${ }^{3}$ Orthopedic Surgery, ${ }^{4}$ Oral Pendulum Medicine, People's Hospital of Zhangqiu, Zhangqiu, Shandong 250200; \\ ${ }^{5}$ Pharmacy Intravenous Admixture Services, The Second Hospital of Shandong University, Jinan, Shandong 250033, P.R. China
}

Received July 7, 2014; Accepted October 22, 2014

DOI: $10.3892 /$ or.2014.3644

\begin{abstract}
This study was mainly focused on the development of a dual-ligand liposomal delivery system for targeting the delivery of paclitaxel (PTX) to lung cancer. The specific ligand peptide HAIYPRH (T7) and the cationic cell-penetrating peptide TAT were connected with phospholipid via a polyethylene glycol (PEG) spacer to prepare the dual-ligand liposomes (T7/TAT-LP-PTX). Physicochemical characterizations of T7/TAT-LP-PTX, such as particle size, $\zeta$ potential, morphology, encapsulation efficiency, and in vitro PTX release, were also evaluated. In the cellular uptake study, the T7/ TAT-LP endocytosed by the A549 cells was 2.26-, 3.48- and 8.56-fold higher than TAT-LP, T7-LP and LP, respectively. The $\mathrm{IC}_{50}$ values of TAT-LP-PTX, T7-LP-PTX and LP-PTX were much higher than those of T7/TAT-LP-PTX, respectively. The homing specificity of T7/TAT-LP was evaluated on the tumor spheroids, which revealed that T7/TAT-LP was more efficaciously internalized in tumor cells than TAT-LP, T7-LP and LP, respectively. Compared to LP, TAT-LP and T7-LP, T7/TAT-LP showed the strongest cell uptake property, and the highest accumulation ability in tumor spheroids in vitro. In the in vivo study, the T7/TAT-LP-PTX exhibited the best inhibitory effect of tumor growth for A549-bearing mice. Collectively, these results suggested that T7/TAT-LP-PTX is a promising drug delivery system for the treatment of lung cancer.

\section{Introduction}

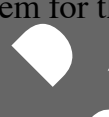

Lung cancer is characterized by uncontrolled cell growth in lung tissues leading to metastasis, invasion of adjacent tissue and infiltration beyond the lungs. Lung cancer was responsible for over 0.15 million deaths in the United States in 2010, with

Correspondence to: Dr Rong-Hua Wang, Department of Cardiothoracic Surgery, People's Hospital of Zhangqiu, Huiquan Road 1920, Zhangqiu, Shandong 250200, P.R. China

E-mail: 529827419@qq.com

Key words: dual-ligand, cell-penetrating peptide, transferrin receptor, liposome over 0.2 million cases registered annually (1). Although surgery is a preferred method of cancer removal, it cannot remove the tissue completely and is required to be supplemented by multidrug chemotherapy and/or radiation as preferred treatment of choice, Etoposide (ETP) and docetaxel (DTX) are the current drugs of choice along with doxorubicin, carboplatin and cisplatin for lung cancer treatment (2). However, these preferred chemotherapeutic agents used in cancer therapy have shown limited therapeutic action.

In the last two decades, liposomal drug delivery systems hold extraordinary potential for delivery of therapeutics to tumor, and various strategies have been used to improve their targeting specificity and cellular uptake. PEGylation has been extensively employed to enhance the accumulation of liposomes in tumor tissues through enhanced permeability and retention (EPR) effects, which was the passive form of targeting (3). In attempts to increase the specificity of interaction between liposomes and tumor cells, recent efforts in the liposome field have focused on the development of active tumor-targeted liposomes, which were modified with specific ligands such as TF (4), folic acid (5), peptides (6-9) or antibodies (10-12), and could selectively recognize and bind to the specific overexpressed receptor in tumor cells, resulting in increased targeting efficiency and less toxicity. Transferrin (TF) receptors are highly expressed in tumor cells (13-15). Peptide T7 (sequenced HAIYPRH) was screened by a phage display system on cells expressing human transferrin receptor (TFR) (16). The high affinity for TFR was comparable to that of TF, with $\mathrm{Kd}$ of w10 nM. Recently, the internalization of the complex formed after T7 binding with TFR was found to be facilitated by endogenous TF (17). Thus, for TFR highly-expressed tumors, $\mathrm{T} 7$ may be a potential ligand for targeting delivery of agents. However, the presence of receptor-targeting moiety alone on PEGylated liposomes limits the cellular uptake of liposomes due to receptor saturation $(18,19)$. Considering that an ideal tumor-targeted drug delivery system should selectively targets delivery drugs to the tumor and delivers the drugs into tumor cells with high efficacy, the receptor saturation should also be overcome. The cell-penetrating peptides (CPPs) conjugated to the surface of liposomes have been widely investigated under in vitro conditions to increase the intracellular delivery of drugs (20). Additionally, the cationic cell-penetrating peptide CPP (TAT) derived from the HIV-1 protein TAT may facilitate 
the intracellular delivery of cargoes with various sizes and physicochemical properties (21-23). Liposomes modified with TAT may deliver the cargoes into cells with high efficiency through an unsaturated and receptor/transporter-independent pathway (24). In the present study, we employed a dual mechanistic approach for targeting TFR on tumor cells and further improving the cellular uptake of the targeted delivery vehicle. We combined the receptor targeting property of $\mathrm{T} 7$ with the enhanced cell uptake effect of TAT to improve the transport of desired cargoes to the tumor.

T7- and TAT-conjugated polyethylene glycol (PEG)modified liposome (T7/TAT-LP) was constructed as a nanoplatform to deliver PTX contrast agent targeting the tumor specifically, yielding T7/TAT-LP-PTX (Fig. 1). The targeting and antitumor efficiency in lung cancer of this contrast agent were evaluated in vitro and in vivo.

\section{Materials and methods}

Materials. Soybean lecithin consisting of 90-95\% phosphatidylcholine and $\mathrm{mPEG}_{2000}-\mathrm{DSPE}$, and $\mathrm{Mal}_{-\mathrm{PEG}_{2000}-\mathrm{DSPE}}$ were purchased from Avanti Lipid (Alabaster, AL, USA). Cholesterol (CHO) was purchased from Chengdu Kelong Chemical Company (Chengdu, China). Rhodamine-PE was purchased from Avanti Lipid. T7 peptide with terminal cysteine (Cys-HAIYPRH) and TAT peptide with terminal cysteine (Cys-AYGRKKRRQRRR) were produced according to the standard solid phase peptide synthesis by Shanghai Jier Bio-Pharmaceutical Co., Ltd. (Shanghai, China). Cell culture plates were purchased from Wuxi NEST Biotechnology Ltd. (Wuxi, China). Other chemicals and reagents were of analytical grade and obtained commercially.

BALB/c male athymic nude mice $(\checkmark 20$ from the Experiment Animal Center (Jinan, China).

Synthesis of TAT-PEG $2000-D S P E$ and T7-PEG $2000-D S P E$. TAT-PEG $_{2000}$-DSPE was synthesized as described previously (25-27), DSPE-PEG 2000 -Mal and Cys-TAT (molar ratio, 1:1.5) were reacted in the mixture of $\mathrm{CHCl}_{3} / \mathrm{MeOH}$ (v:v, 2:1) with gentle stirring at room temperature overnight. After thin layer chromatography (TLC) showed the disappearance of DSPE-PEG ${ }_{2000}-\mathrm{Mal}$, the mixture was evaporated under vacuum, the residue was redissolved by $\mathrm{CHCl}_{3}$, and the insoluble material was filtered, the supernatant (DSPE-PEG ${ }_{2000}-\mathrm{TAT}$ ) was evaporated again under vacuum and stored at $-20^{\circ} \mathrm{C}$ until use.

The T7-PEG ${ }_{2000}-\mathrm{DSPE}$ was synthesized according to literature with a modest modification $(16,28)$. T7 was conjugated with DSPE-PEG ${ }_{2000}-\mathrm{BTC}$ in $0.01 \mathrm{M}$ isotonic HEPES buffer (pH 7.5) under the reaction conditions of $4 \mathrm{~h}$ at $4^{\circ} \mathrm{C}$, gentle stirring and 1:2 molar ratio of peptides to DSPE-PEG ${ }_{2000}-\mathrm{BTC}$. The reaction was traced by TLC until the peptide was completely consumed. The mixture was then dialyzed against water, and lyophilized. The resulting conjugate DSPE-PEG ${ }_{2000}-\mathrm{T} 7$ was then used to prepare liposomes without further purification.

Preparation ofliposomes. TAT and T7 co-modified PTX-loaded liposomes (T7/TAT-LP-PTX) were prepared by thin film hydration methods $(29,30)$. Briefly, SPC, CHO, PTX $(10 \%$ of the SPC + CHO weight), DSPE-PEG ${ }_{2000}$, DSPE$_{-P_{20}}-\mathrm{TAT}$ and DSPE-PEG $2000-\mathrm{T} 7$ were dissolved in chloroform (total molar ratio of phospholipid and $\mathrm{CHO}$ derivatives was 3:2, molar ratio of DSPE-PEG ${ }_{2000}$, DSPE-PEG $_{2000}$-TAT and DSPE$\mathrm{PEG}_{2000}-\mathrm{T} 7$ was 9.5:4.5:0.5). Chloroform was then evaporated by rotary evaporation and residual organic solvent was removed in vacuum overnight. The thin film was hydrated in phosphatebuffered saline (PBS, pH 7.4) for $1 \mathrm{~h}$ at $37^{\circ} \mathrm{C}$, followed by an intermittent probe sonication for $50 \mathrm{sec}$ at $100 \mathrm{~W}$.

Rhodamine-labeled liposomes were prepared as the T7/TAT-LP-PTX with the SPC being replaced by the rhodamine-PE. The final concentration of rhodamine-PE was $10 \mu \mathrm{g} / \mathrm{ml}$.

\section{Characterization of liposomes}

Size and $\zeta$ potential measurements. The size and zeta potential of the liposomes were measured by a dynamic light scattering detector (Zetasizer Nano ZS90; Malvern Instruments, Ltd., UK)

Drug encapsulation efficiency (EE) and drug-loading efficient. The free PTX was removed by passing through a Sephadex G -50 column. The amount of PTX encapsulated in the liposome was measured by high-performance liquid chromatography (HPLC, Agilent LC1100). A reversed phase Inertsil ${ }^{\circledR}$ ODS -3 column $(150-4.6 \mathrm{~mm}$, pore size $5 \mathrm{~mm}$; GL Sciences Inc., Tokyo, Japan) was used. Liposomes were dissolved in $1 \mathrm{ml}$ DCM. After evaporating DCM, $3 \mathrm{ml}$ mobile phase (50:50 v/v acetonitrile/water solutions) was added to dissolve the drugs. The solution was then filtered by $0.45 \mathrm{~mm}$ PVDF syringe filter for HPLC analysis. The column effluent was detected at $227 \mathrm{~nm}$ with a UV/VIS detector. EE (\%) was calculated as: (amount of drug encapsulated in LP/initial amount of drug used in the fabrication of LP) x100\%.

In vitro stability of liposomes in serum. To demonstrate the erum stability of liposomes, particle sizes and turbidity variations were monitored in the presence of fetal bovine serum (FBS) (31,32). Briefly, liposomes were mixed with an equal volume of $\mathrm{FBS}$ at $37^{\circ} \mathrm{C}$ with gentle agitation at $30 \mathrm{rpm}$. At predetermined time-points (1, 2, 4, 8 and $24 \mathrm{~h}), 200 \mathrm{ml}$ of the sample was pipetted out and onto a 96 -well plate to measure the transmittance at $750 \mathrm{~nm}$ using a microplate reader (Varioskan Flash; Thermo Scientific, Waltham, MA, USA) and another $200 \mathrm{ml}$ was diluted to $1 \mathrm{ml}$ with $5 \%$ glucose solution for the particle size measurements by Malvern Zetasizer Nano ZS90 Instrument (Malvern Instruments Ltd.).

In vitro drug release. In vitro PTX release study was conducted using dialysis method under sink conditions (33). An aliquot of each PTX-loaded liposome $(0.1 \mathrm{ml})$ or free PTX was placed into the dialysis tube (MWCO 8000) and tightly sealed. The dialysis tubes were immersed into $100 \mathrm{ml}$ PBS (pH 7.4) containing $0.1 \%$ (v/v) Tween-80 and were incubated at $37^{\circ} \mathrm{C}$ for $24 \mathrm{~h}$ with mild oscillating at $50 \mathrm{rpm}$. At predetermined time-points, $0.1 \mathrm{ml}$ release medium was sampled and replaced with equal volume of fresh release medium. The samples were diluted with acetonitrile and the concentrations of PTX were determined by HPLC.

In vitro cellular uptake. A549 cells were grown in RPMI1640 medium (Gibco-Life Technologies, Carlsbad, CA, USA) contains $10 \% \mathrm{FBS}, 100 \mu \mathrm{g} / \mathrm{ml}$ streptomycin, and $100 \mathrm{U} / \mathrm{ml}$ penicillin. The cells were maintained at $37^{\circ} \mathrm{C}$ in a humidified incubator with $5 \% \mathrm{CO}_{2}$. 

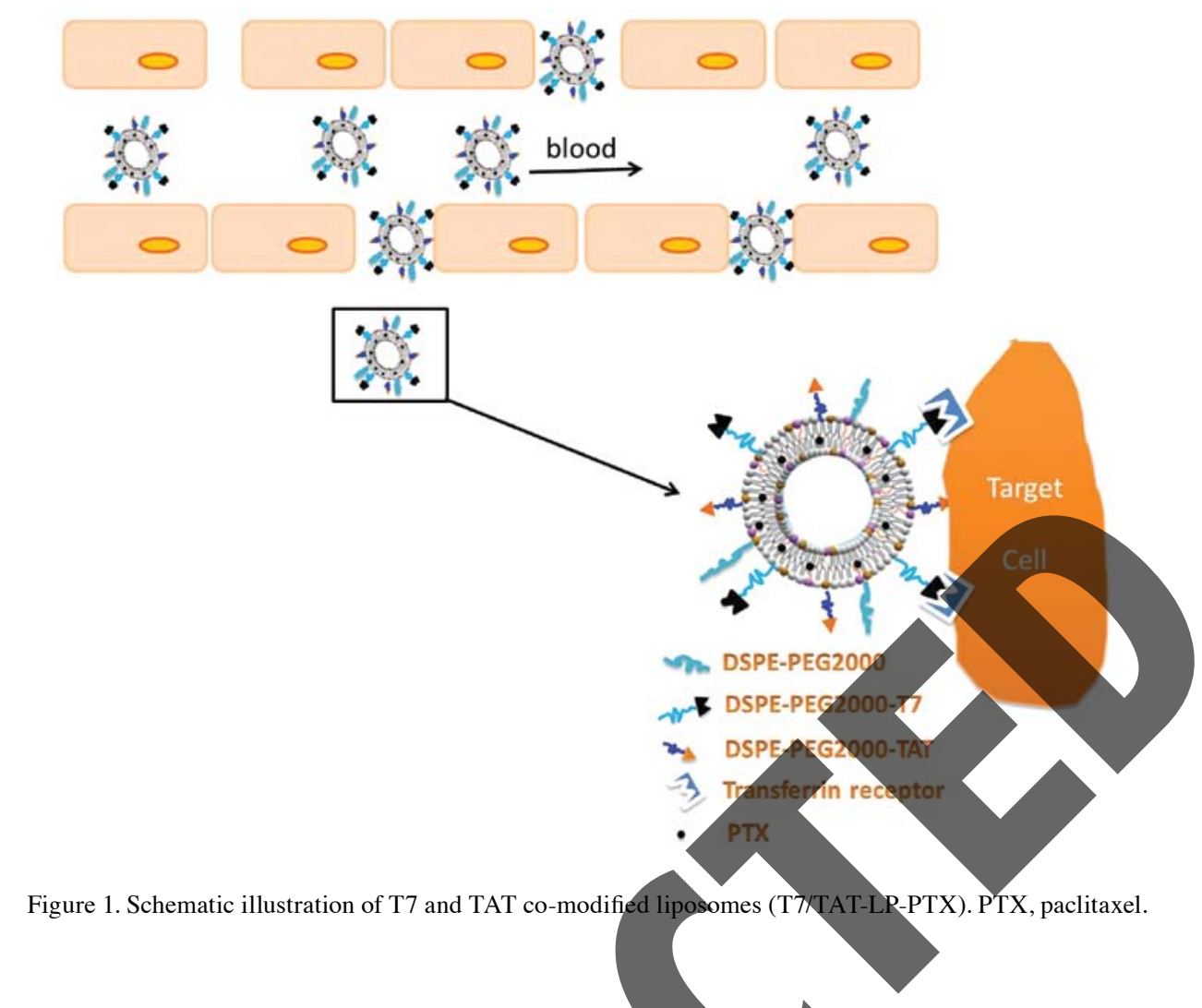

For quantitative study, A549 cells a (American Type Culture Collection; Manassas, VA, USA) were haryested with $0.125 \%$ Gibco Trypsin-EDTA solution (Invitrogen-Life respectively, were added. To study the effect of temperature on the cellular uptake, the cells were incubated at 37 and $4^{\circ} \mathrm{C}$. The inhibitor-containing culture media were discarded and Technologies) and seeded into 6-well assay plates-(Corning thodamine-labeled liposome, containing culture media, was Inc., Corning, NY, USA) at $5 \times 10^{5}$ viable cells/well. After $24 \mathrm{~h}, \quad$ applied for $4 \mathrm{~h}$ incubation. The cells were treated as described it was replaced with fresh serum-free medium, and then the in the quantitative study, and the fluorescence intensity was prepared rhodamine-labeled liposomes were added to the determined by flow cytometer $\left(\right.$ Cytomics $^{\mathrm{TM}}$ FC 500; Beckman cells. The final lipid concentration was $0.2 \mathrm{mg} / \mathrm{ml}$. After incu- $>$ Coulter, Miami, FL, USA).

bation for $4 \mathrm{~h}$, the medium was discarded and the cells were washed three times with cold PBS. The cells were harvested and washed with cold PBS, centrifuged at 4,000 rpm and re-suspended three times. The cellular uptake efficiency was determined by FACS.

For the qualitative study, A549 cells were harvested with $0.125 \%$ Gibco Trypsin-EDTA solution (Invitrogen) and seeded in Lab-Tek coverglass chambers (Nagle Nunc, IL, USA) with RPMI-1640 at a concentration of $5 \times 10^{3}$ viable cells/chamber. The cells were incubated overnight and were subsequently incubated with rhodamine-labeled liposomes in the RPMI-1640 (concentration of $10 \mu \mathrm{g} / \mathrm{ml}$ ) at $37^{\circ} \mathrm{C}$. After $4 \mathrm{~h}$, the cells were washed three times with cold PBS and fixed with 4\% paraformaldehyde for $20 \mathrm{~min}$. The cells were washed twice with cold PBS. The nuclei were stained by incubating with DAPI (Roche, Mannheim, Germany) for another $10 \mathrm{~min}$. The cell monolayer was washed three times with PBS and observed by confocal laser scanning microscopy (Leica, Munich, Germany).

Identification of cellular uptake pathways. To study the effect of different inhibitors on the cellular uptake of T7/ TAT-LP, the cells were pre-incubated with different inhibitors for $30 \mathrm{~min}$ at $37^{\circ} \mathrm{C}$. Poly-lysine $(800 \mathrm{mg} / \mathrm{ml})$, amiloride $(1.48 \mathrm{mg} / \mathrm{ml})$, chlorpromazine $(20 \mathrm{mg} / \mathrm{ml})$, filipin $(5 \mathrm{mg} / \mathrm{ml})$, free T7 peptide $(5 \mathrm{mg} / \mathrm{ml})$ and free TAT peptide $(5 \mathrm{mg} / \mathrm{ml})$,

In vitro cytotoxicity and anti-proliferation assay. Comparison of in vitro cytotoxicity and tumor cell proliferation assay of various formulations was performed on A549 cells using SRB colorimetric assay. Briefly, A549 cells $\left(4 \times 10^{3}\right.$ cells) were seeded in 96-well plates and incubated overnight. The cells were exposed to serial concentrations of different PTX formulations in the culture medium for $48 \mathrm{~h}$ at $37^{\circ} \mathrm{C}$. Subsequently, cells were fixed with trichloroacetic acid, washed, and stained with SRB. Absorbance was measured at $540 \mathrm{~nm}$ using a 96-well plate reader (Bio-Rad Laboratories, Hercules, CA, USA). Dose-response curves were generated, and the concentrations of drug resulting in $50 \%$ cell killing $\left(\mathrm{IC}_{50}\right)$ were calculated by Origin 7.0 (OriginLab, Northampton, MA, USA).

Evaluation of tumor spheroid penetration. To prepare the three-dimensional tumor spheroids, A549 cells were seeded at a density of $2 \times 10^{3}$ cells $/ 200 \mu 1$ per well in 96 -well plates coated with $80 \mu 1$ of a $2 \%$ low-melting-temperature agarose. Seven days after the cells were seeded, tumor spheroids were treated with $10 \mu \mathrm{g} / \mathrm{ml}$ rhodamine-labeled liposome. After $4 \mathrm{~h}$ of incubation, the spheroids were rinsed three times with ice-cold PBS and fixed with 4\% paraformaldehyde for $30 \mathrm{~min}$. The spheroids were transferred to glass slides and covered by glycerophosphate. Fluorescent intensity was observed by laser scanning confocal microscopy (Leica). 
Table I. Characteristics of PTX-loaded LP, TAT-LP, T7-LP and T7/TAT-LP ( $\mathrm{n}=3$ ).

\begin{tabular}{llcrr}
\hline Group & $\begin{array}{c}\text { Particle } \\
\text { size }(\mathrm{nm})\end{array}$ & Polydispersity & $\begin{array}{c}\zeta \text { potential } \\
(\mathrm{mV})\end{array}$ & $\begin{array}{r}\text { Encapsulation } \\
\text { efficiency }(\%)\end{array}$ \\
\hline LP-PTX & $101 \pm 10.8$ & 0.167 & $-2.52 \pm 1.44$ & $88.45 \pm 1.23$ \\
TAT-LP-PTX & $109 \pm 9.4$ & 0.120 & $21.36 \pm 1.37$ & $87.24 \pm 1.25$ \\
T7-LP-PTX & $110 \pm 6.7$ & 0.132 & $3.67 \pm 1.45$ & $86.65 \pm 1.37$ \\
T7/TAT-LP-PTX & $106 \pm 7.5$ & 0.138 & $23.74 \pm 1.08$ & $85.47 \pm 1.55$ \\
\hline
\end{tabular}

PTX, paclitaxel.

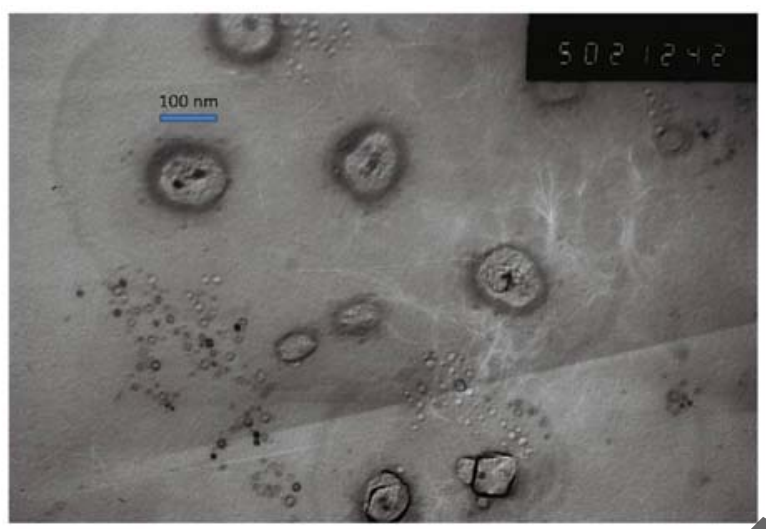

Figure 2. A transmission electron microscopy (TEM) image shows the ture of the T7/TAT-LP-paclitaxel (PTX).

\section{Results and Discussion}

Characterization of the liposome

Particle size, size distribution, drug EE and drug-loading efficient. The shape and surface morphology of T7/TAT-LP-PTX investigated was observed via transmission electron microscopy (Fig 2). The electron microscope results showed that the liposomes all exhibited uniform spherical appearance. The average diameter of the conventional PTX-loaded liposomes was $100 \mathrm{~nm}$ with a PDI of 0.150 (Table I). The drug EE of the nanoparticles was crucial to justify their clinical applications (34). The PTX EE of LP, TAT-LP, T7-LP and T7/TAT-LP was $>85 \%$. Table I shows the EE of the four types of liposome formulations. Obviously, such a formulation system demonstrates the prospect for a practically useful drug delivery carrier with appropriate size, stability and drug-loading capacity.

Stability of PTX-loaded liposomes in the presence of FBS. Liposomal particle stability against physiological condition is In vivo tumor growth inhibition study. The lung cancer nude mouse xenograft models were established by injecting A549 cells $\left(1 \times 10^{7}\right.$ cells/animal, subcutaneous injection) into the back of 4- to 6-week-old BALB/c male athymic nude mice. Tumor volume $\left(\mathrm{mm}^{3}\right)$ was measured with vernier caliper. Fifty nude mice with lung cancer xenograft models were divided into five groups. When the tumors reached $100-200 \mathrm{~mm}^{3}$, the mice were administrated with PBS, free PTX, TAT-LP-PTX, T7-LP-PTX and T7/TAT-LP-PTX, respectively. The drugs were administered once every other day (totally $10 \mathrm{mg} / \mathrm{kg}$ ) and the tumor volumes were measured. The tumor inhibition rate was calculated using the formula: Tumor inhibition rate $=\left(1-\mathrm{V}_{\mathrm{t}} /\right.$ $\left.\mathrm{V}_{0}\right) \times 100 \%$, where $\mathrm{V}_{\mathrm{t}}$ was the tumor volume of the treated mice and $\mathrm{V}_{0}$ was the initial tumor volume of the untreated mice.

In vivo imaging. The DIR-loaded liposomes were utilized, as previously described, to investigate the distribution of liposomes in nude mouse-bearing A549 cells. The DIR-loaded liposomes were injected into nude mouse-bearing A549 cells via intravenous administration, and then the in vivo fluorescence imaging was performed by IVIS Spectrum system (Caliper Life Sciences, Hopkington, MA, USA).

Statistical analysis. Data are presented as means \pm SD. Analysis of variance (ANOVA) was used to determine the variance of the whole values in each group. Statistical significance was evaluated using the Student's t-test for comparisons of the experimental groups. prerequisite for the further application in vivo, thus 50\% FBS was employed to mimic the in vivo situation. Particle sizes and transmittance variations as important parameters were monitored in this study to examine the serum stability of liposomes. The particle sizes and transmittance hardly changed for the liposomes over $24 \mathrm{~h}$, indicating that there was no aggregation in the presence of serum (Fig. 3).

In vitro drug release. The in vitro release of PTX from the liposomes was investigated. Fig. 4 shows the release profile of the four groups. Compared with the rapid release of free PTX, the four liposome groups exhibited a similar and sustained release manner and no initial burst release was observed.

Cellular uptake. A549 cell uptake of rhodamine-labeled TAT-LP, T7-LP, LP and TAT/T7-LP at different capacities was observed (Fig. 5). The T7/TAT-LP endocytosed by the A549 cells was 2.26-, 3.48- and 8.56-fold higher than TAT-LP, T7-LP and LP, respectively. The T7-LP stimulated the uptake by 2.45 -fold compared with the LP, and the uptake of T7/TAT-LP increased 3.48-fold compared with the TAT-LP, indicating that the T7 motif had the ability to recognize and target TF receptors expressed on the cell surface. Moreover, the modification of TAT exhibited a synergistic effect with T7 on the cellular uptake of liposomes in TF-expressing cells, suggesting that after the recognition of TF by the T7 motif, the TAT further enhanced the internalization of liposomes. The cellular uptake of liposomes was studied by confocal microscopy. The cellular uptake of LP was set as a control 

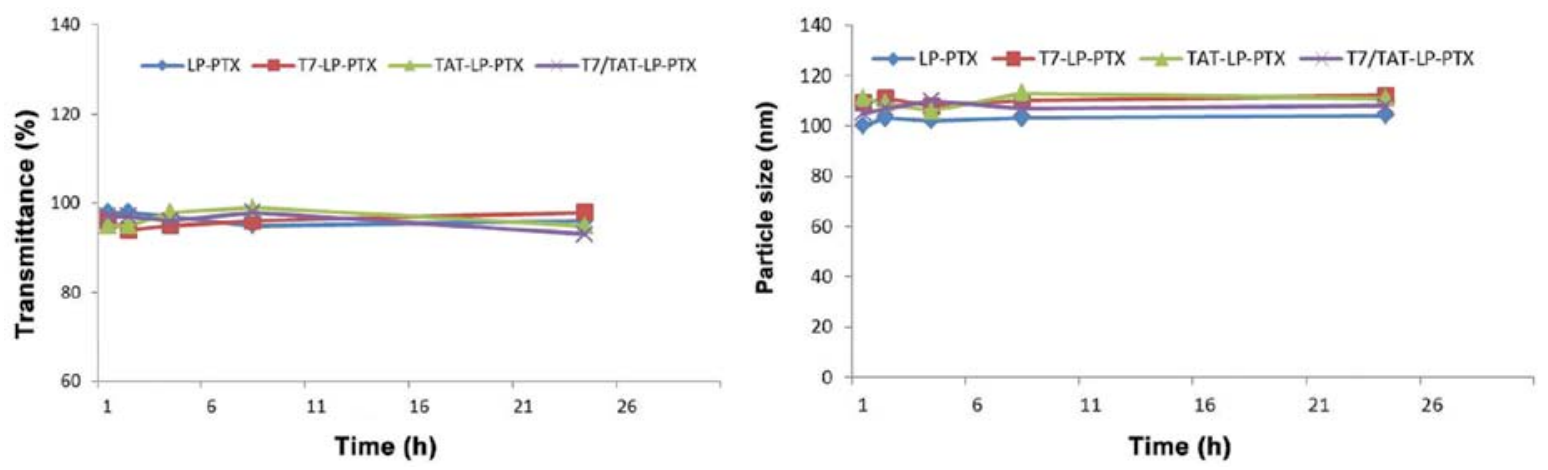

Figure 3. (A) Variations in particle sizes of liposomes in 50\% fetal bovine serum (FBS). (B) Variations in turbidity (represented by transmittance) of liposomes in $50 \%$ FBS $(n=3$, mean \pm SD).

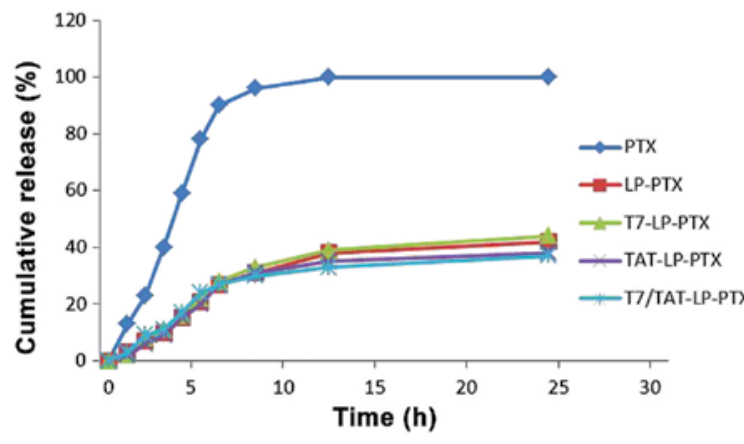

Figure 4. The paclitaxel (PTX) release profiles of free PTX, LP-PTX TAT-LP-PTX, T7-LP-PTX and T7/TAT-LP-PTX in phosphate-buffered saline (PBS) over $24 \mathrm{~h}(\mathrm{n}=3$, mean $\pm \mathrm{SD})$.

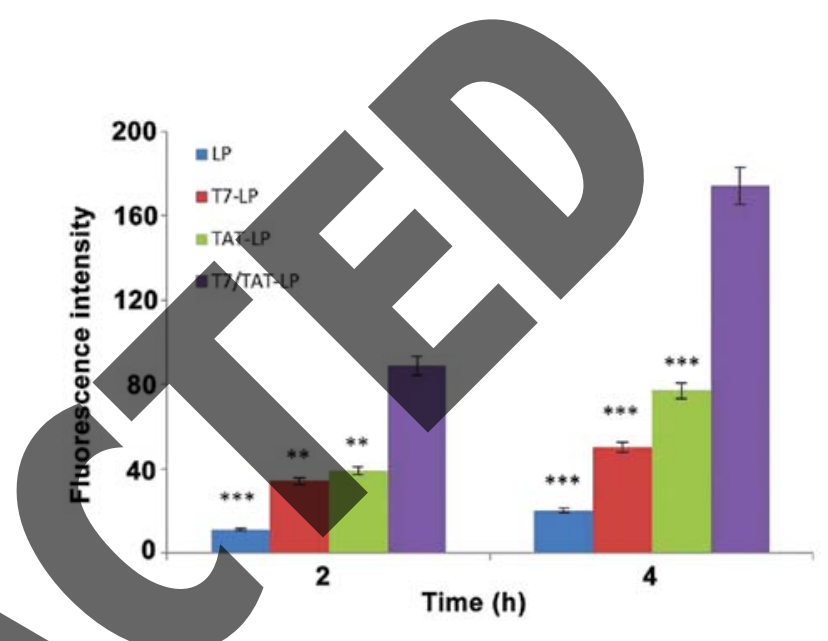

Figure 5. Measurement of in vitro uptake of rhodamine-labeled LP, T7-LP, TAT-LP and T7/TAT-LP by A549 cells. Data are presented as the mean $\pm \mathrm{SD}$, $=3 . \quad \mathrm{P}<0.01$ and ${ }^{* * * *} \mathrm{P}<0.001$.

(Fig. 6). Compared to the control, the T7 liposomes showed a weak fluorescence signal in A549 cells, indicating that the TF-mediated cellular uptake was limited. By contrast, much higher cellular uptake was observed for the TAT liposomes in A549 cells, suggesting that cell-penetrating peptide TAT had the ability to mediate cellular uptake of liposomes. The T7/TAT-LP resulted in stronger fluorescence signals than the TAT-LP and T7-LP in A549 cells, indicating that the dual ligands exhibited a synergistic effect on the cellular uptake of liposomes in TF receptor-expressing cells. These results showed that the synergistic effect of the dual-ligand liposomes was correlated with the conformation of the two ligands.

Identification of cellular uptake pathways. To examine the endocytosis pathways for T7 and TAT co-modified liposomes, an endocytosis inhibition assay was conducted. To determine the possible involvement of different endocytic pathways in the cellular uptake of liposomes in A549 cells, several classical inhibitors of endocytosis were used, and the fluorescence of cells treated with the different liposomal formulations without any inhibitor was set as $100 \%$ and as the control. The uptake of TAT-LP, T7-LP and TAT/T7-LP was decreased by $~ 89,78$ and $92 \%(\mathrm{P}<0.001)$ in comparison with the control at $4^{\circ} \mathrm{C}$ due to the downregulated cell metabolism, suggesting that the cellular uptake of liposomes was dependent on temperature (Fig. 7). Cell treatment with the energy inhibitor $\mathrm{NaN}_{3}$ did not significantly change the cellular uptake of T7-LP. However, the cellular uptake of TAT-LP and TAT/T7-LP was decreased by $\sim 14 \%(\mathrm{P}<0.05)$, indicating that the cellular uptake of TAT-LP

and TAT/T7-LP was dependent on energy. A decrease of $74 \%$ $(\mathrm{P}<0.01)$ and $67 \%(\mathrm{P}<0.01)$ in the cellular uptake of TAT-LP and TAT/T7-LP was observed after incubation in the presence of poly-L-lysine (PPL), which was a positively charged amino acid polymer that had the ability to bind with the negatively charged cell membrane, suggesting that the uptake of TAT-LP and TAT/T7-LP was influenced by the charges of liposomes. It was found that the TAT sequence contained many basic amino acids which had strong cationic properties, thus electrostatic interactions were generated between TAT and the negatively charged cell membrane. Therefore, the cellular uptake of TAT/T7-LP was mainly influenced by the cationic nature of the TAT motif. The uptake of T7-LP and TAT-LP was decreased by $\sim 15 \%(\mathrm{P}<0.05)$ and $22 \%(\mathrm{P}<0.01)$ after incubation with colchicine. Colchicine is known to inhibit the formation of microfilaments and microtubules (22). Therefore, colchicine exerted an effect on macropinocytosis-mediated uptake. The small effect of colchicine on the uptake of T7-LP and TAT-LP showed that macropinocytosis was presumably involved to a lesser extent. At the same time, by preventing the recycling of clathrin and hindering endocytosis through clathrin-dependent mechanisms with the cationic amphiphilic drug chlorpromazine, a significant decrease (38 and 34\%, $\mathrm{P}<0.01)$ in the cellular uptake of T7-LP and TAT-LP was observed in the presence of chlorpromazine, suggesting that the clathrin-dependent pathway was involved in the internalization of T7-LP and TAT-LP. The cellular uptake of all liposomes did 

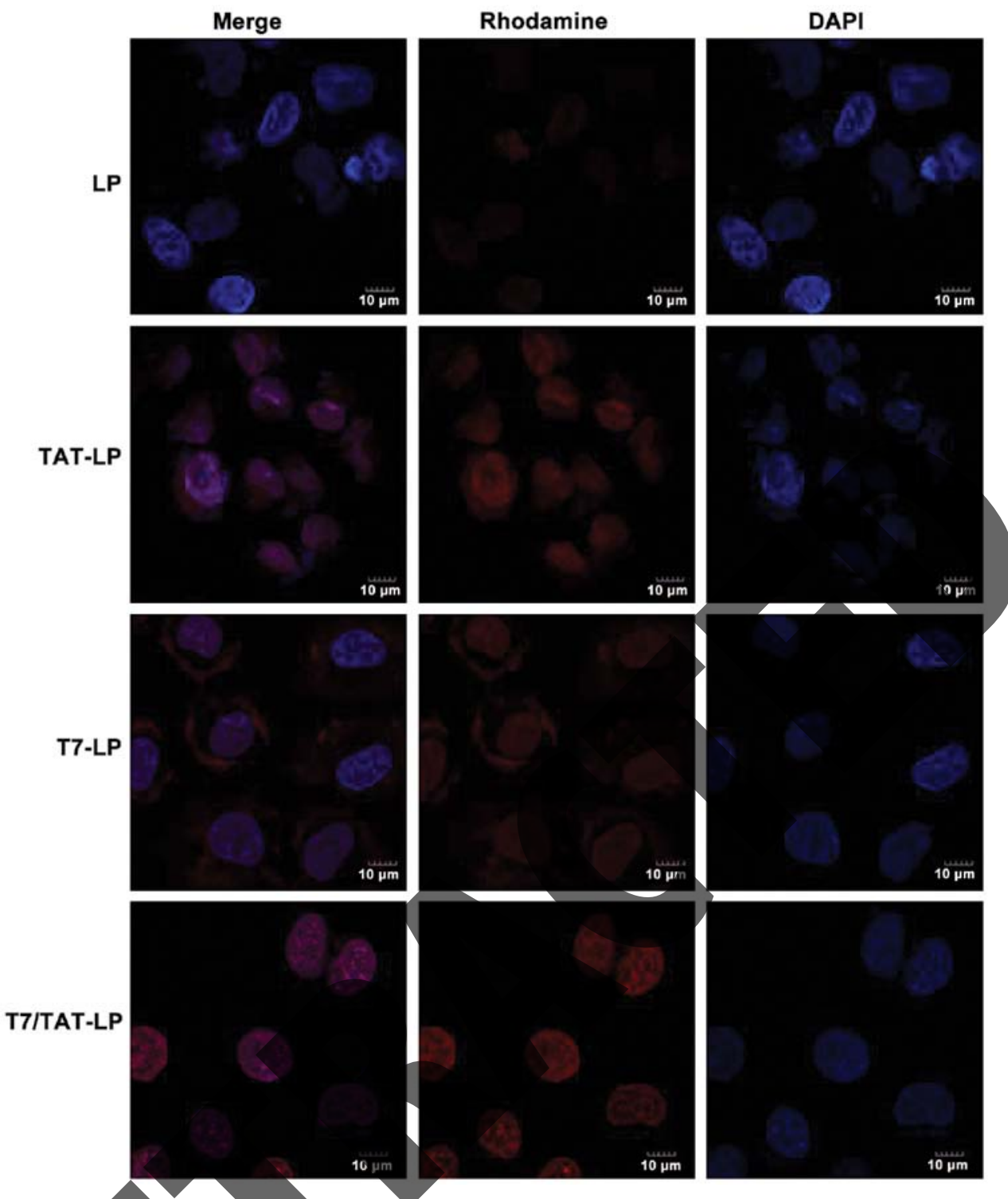

Figure 6. Confocal laser scanning microscopy images show the internalization of fluorescent liposomes in cells ( $4 \mathrm{~h}$ incubation). Column 1 , merged channels of rhodamine and DAPI channels; column 2, rhodamine channels showing the red fluorescence from rhodamine-labeled liposomes distributed in cytoplasm; column 3, DAPI channels showing the blue fluorescence from DAPI-stained nuclei.

Table II. Cytotoxicity against A549 of various PTX formulations in vitro after $48 \mathrm{~h}$ incubation.

\begin{tabular}{lc}
\hline Formulations & $\mathrm{IC}_{50}$ on A549 cells, $\mu \mathrm{g} / \mathrm{ml}$ \\
\hline PTX & 0.0680 \\
LP-PTX & 0.03843 \\
T7-LP-PTX & 0.01240 \\
TAT-LP-PTX & 0.00840 \\
TAT/T7-LP-PTX & 0.00430 \\
\hline
\end{tabular}

PTX, paclitaxel.

not significantly change with the caveolae-dependent endocytosis inhibitor filipin. These results suggested that the cellular uptake of these liposomes was determined by the combination of various endocytic pathways. The cellular uptake mechanism of TAT/T7-LP was similar to that of TAT-LP, indicating that the TAT dominated in the cellular uptake process of TAT/T7-LP. Free TAT peptide did not reduce the cellular uptake of TAT-LP and TAT/T7-LP, suggesting that there was no competitive inhibition and TAT-LP was not internalized into the cells via specific receptors for the TAT peptide sequence. The uptake of T7-LP and TAT-LP was decreased by $~ 64 \%(\mathrm{P}<0.001)$ and $31 \%(\mathrm{P}<0.01)$ after incubation with Free $\mathrm{T} 7$ peptide, suggesting that there was competitive inhibition and T7-LP was internalized into the cells via specific receptors.

In vitro cytotoxicity and anti-proliferation assay. The cytotoxicity effect against A549 cells of various PTX formulations are summarized in Table II. Modification with T7 and TAT resulted in improved efficacy for PTX-loaded liposomes.

Evaluation of tumor spheroid penetration. In many solid tumors, there are hypoxic and avascular tumor regions (35-37). Due to the poor permeation of delivery systems, the amount 


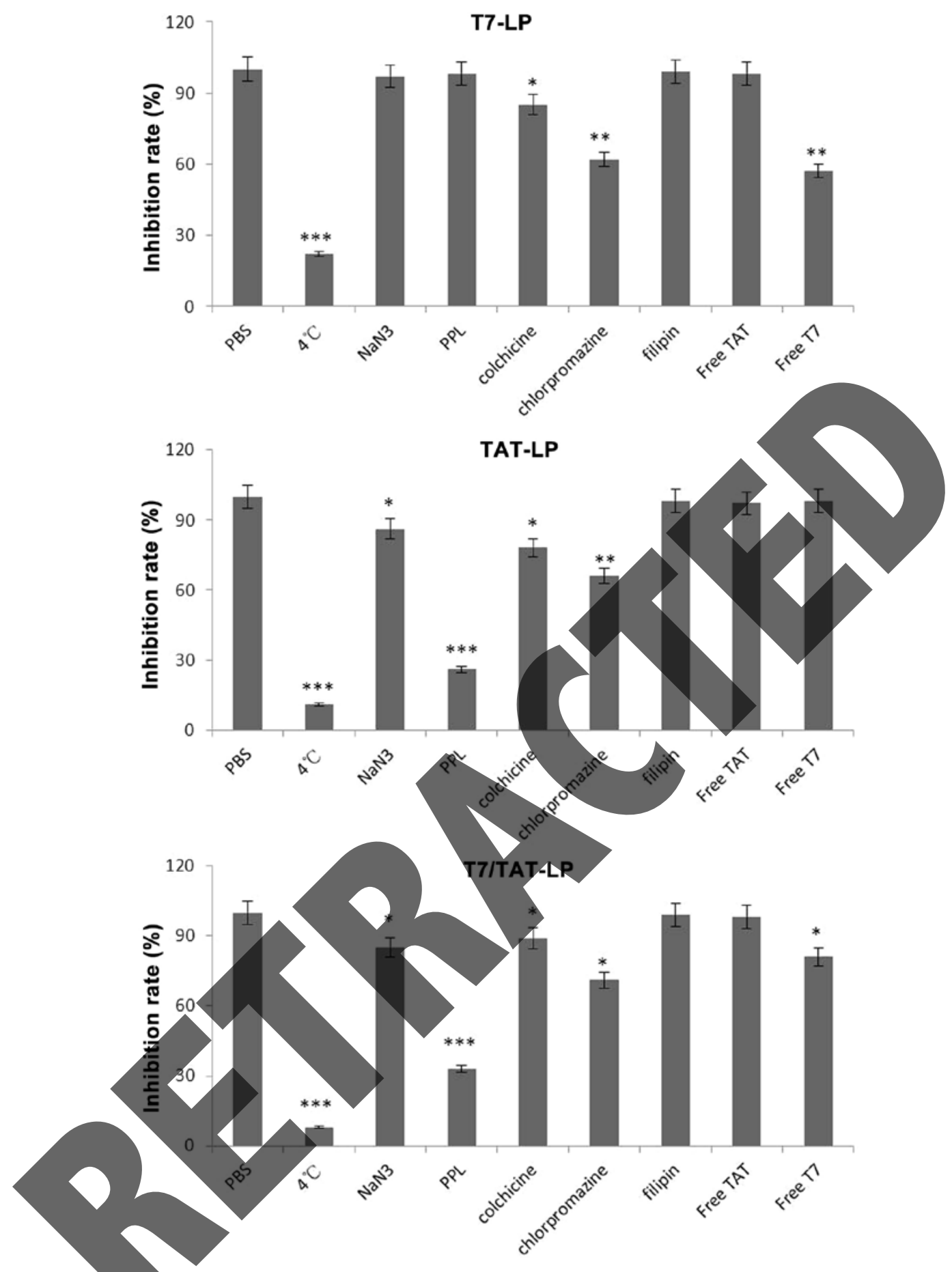

Figure 7. Effects of endoeytosis inhibitors on the cellular uptake of liposomes in A549 cells. The data are presented as the mean $\pm \mathrm{SD}(\mathrm{n}=3)$. ${ }^{*} \mathrm{P}<0.05{ }^{, * *} \mathrm{P}<0.01$ and ${ }^{* * *} \mathrm{P}<0.001$.

of drug reaching inside the solid tumors was low. The tumor spheroids were able to imitate the in vivo status because the tumor spheroids were free of blood vessels (37-39). To evaluate the effects of the solid tumor penetration of the multistage liposomes in vitro, multicellular 3D tumor spheroids, which have been proposed as models of intermediate complexity between monolayer cultures and xenografts because of their similarities to in vivo avascular tumor tissues, were observed by confocal microscopy. Fig. 8 shows confocal laser scanning microscopic images of 3D tumor spheroids after $4 \mathrm{~h}$. LP almost entirely lacked efficient penetration of A549 spheroids, indicating that the liposomes without modification had a very weak penetration. For the T7-LP and TAT-LP, much higher fluorescence was observed primarily on the periphery of tumor spheroids. However, apparently enhanced fluorescence of the TAT/T7-LP was observed, suggesting that solid tumor penetration was enhanced by the synergistic effect of T7 and TAT.

In vivo tumor growth inhibition study. The antitumor effects of PTX-loaded formulations were evaluated on A549 tumor xenografts models on BALB/C nude mice. Compared with the rapid tumor volume growth in PBS group, other groups 

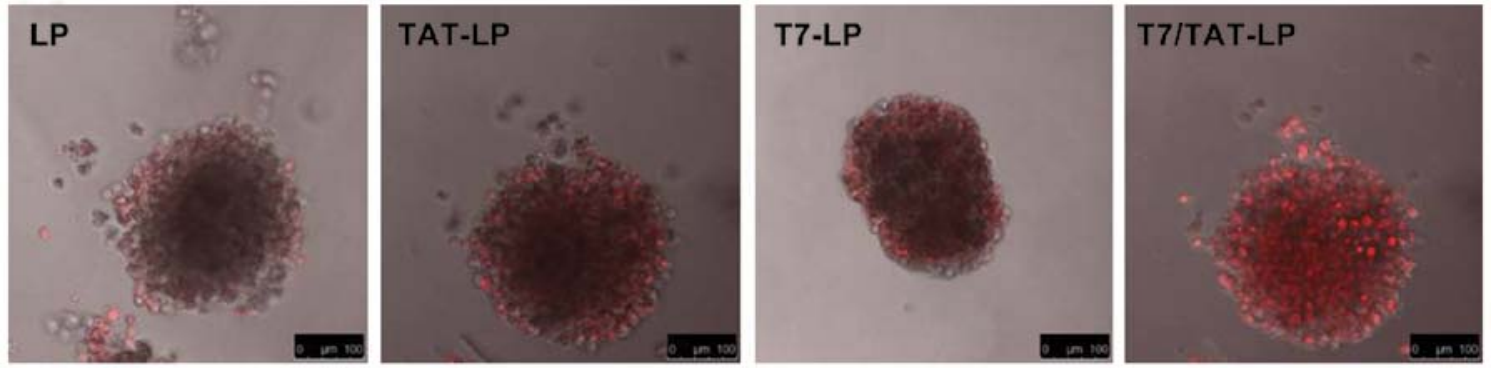

Figure 8. Penetration of rhodamine-labeled LP, T7-LP, TAT-LP and T7/TAT-LP throughout A549 tumor spheroids at $4 \mathrm{~h}$.

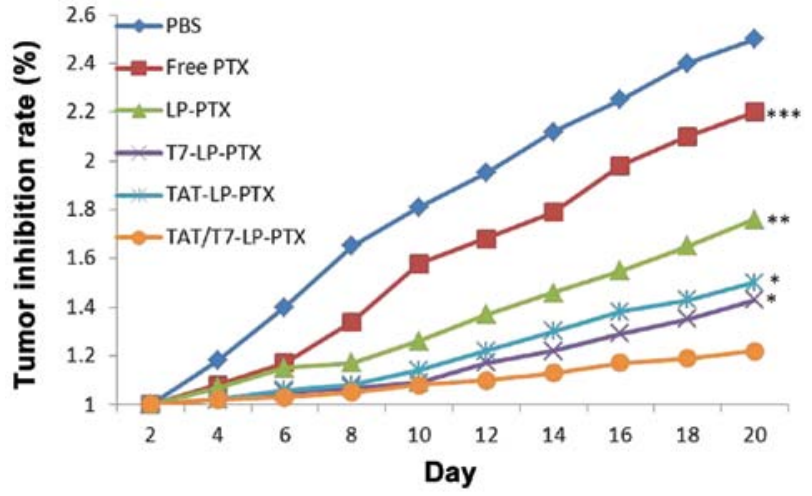

Figure 9. In vivo antitumor effect of different paclitaxel (PTX) formulations in tumor-bearing mice. ${ }^{*} \mathrm{P}<0.05,{ }^{* *} \mathrm{P}<0.01$ and ${ }^{* * *} \mathrm{P}<0.001$.
NIR imaging. The in vivo biodistribution and tumor accumulation profiles of DIR-loaded liposomes were clearly visualized by monitoring the whole body NIRF intensity in the subcutaneous xenograft bearing nude mouse model (Fig. 10). Tumor accumulation was the highest for TAT/T7-LP. These results suggested that the TAT/T7-LP efficiently targeted solid tumors and decreased non-specific accumulation in healthy organs such as livers, lungs and kidneys Control animals injected with PBS produced no fluorescence signals, which confirmed that the observed fluorescence signal was essentially from the liposomes.Preliminary studies (unpublished) have demonstrated that the passive accumulation of liposomes reached maximum in tumor tissue between 24 and $48 \mathrm{~h}$, which provided sufficient time for liposomes to accumulate at the tumor site. The results of serum stability and biodistribution suggested that the liposome was capable of increasing the stability of liposomes, and ultimately, the TAT/T7-LP obtained remarkable accumulation in the tumor region.

In conclusion, in this study, we successfully developed the dual-ligand liposomes modified with the specific ligand T7 motif and non-specific TAT. This liposomal delivery system possessed increased cellular uptake efficiency and targeting specificity in A549 cells whose TFR expression levels were high, and achieved an efficient synergistic targeted delivery of payload into tumor cells in A549 tumor bearing nude mice, ultimately achieving excellent therapeutic efficacy on inhibition compared with other liposomes.

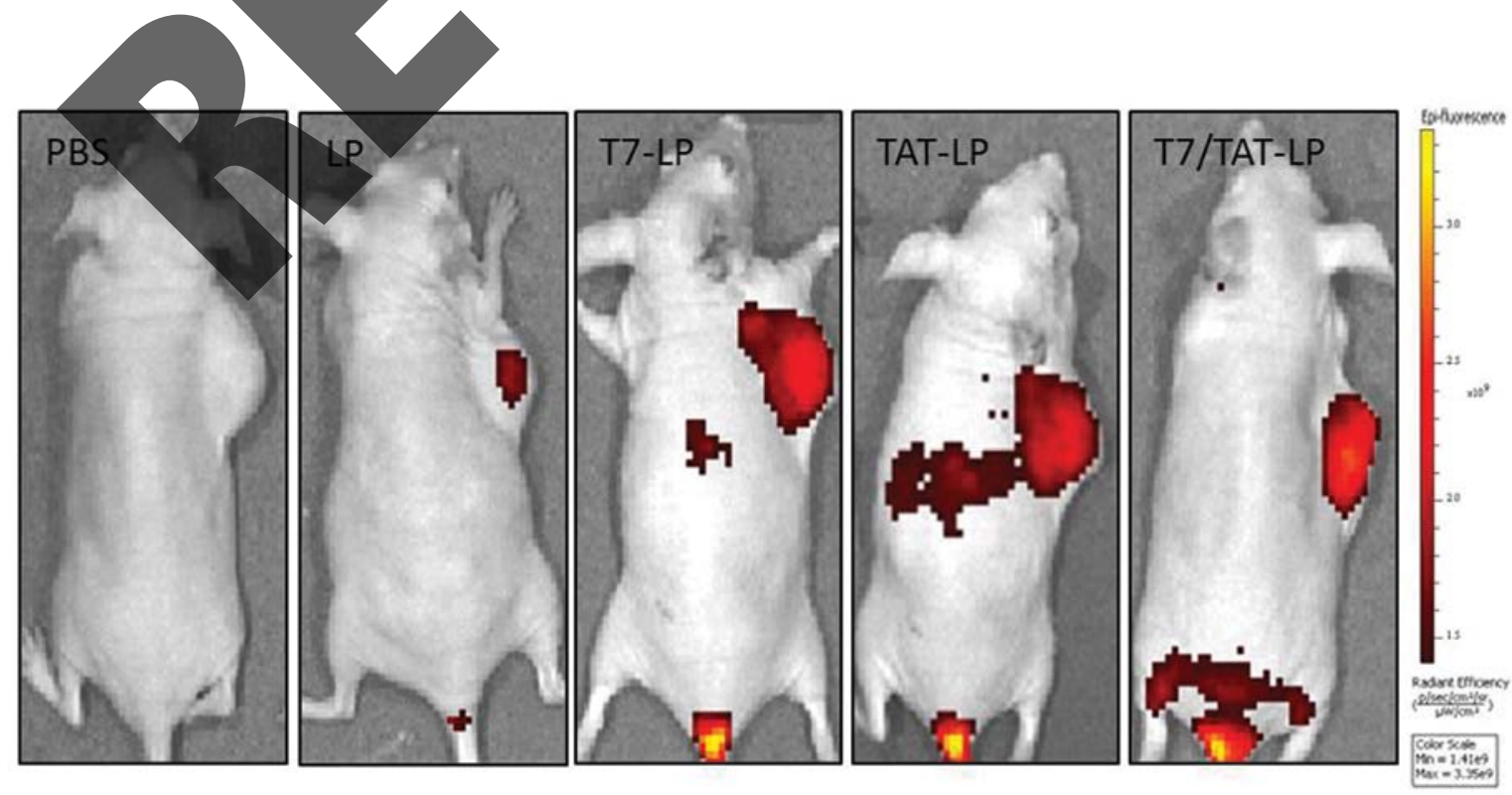

Figure 10. The in vivo image of the mice that were anesthetized at $24 \mathrm{~h}$ after intravenous injection of different types of DIR-loaded liposomes, respectively. 
tumor-bearing mice. The findings of this study suggest that the T7- and TAT-modified liposomes are a potential antitumor drug delivery system.

\section{References}

1. Jinturkar KA, Anish C, Kumar MK, Bagchi T, Panda AK and Misra AR: Liposomal formulations of etoposide and docetaxel for p53 mediated enhanced cytotoxicity in lung cancer cell lines. Biomaterials 33: 2492-2507, 2012.

2. Sengupta S, Tyagi P, Velpandian T, et al: Etoposide encapsulated in positively charged liposomes: pharmacokinetic studies in mice and formulation stability studies. Pharmacol Res 42: 459-464, 2000.

3. Maeda H: Macromolecular therapeutics in cancer treatment: the EPR effect and beyond. J Control Release 164: 138-144, 2012.

4. Tang J, Zhang L, Liu Y, et al: Synergistic targeted delivery of payload into tumor cells by dual-ligand liposomes co-modified with cholesterol anchored transferrin and TAT. Int J Pharm 454 31-40, 2013.

5. Shmeeda H, Amitay Y, Gorin J, et al: Delivery of zoledronic acid encapsulated in folate-targeted liposome results in potent in vitro cytotoxic activity on tumor cells. J Control Release 146: 76-83, 2010.

6. Zhang Q, Tang J, Fu L, et al: A pH-responsive $\alpha$-helical cell penetrating peptide-mediated liposomal delivery system. Biomaterials 34: 7980-7993, 2013.

7. Jiang X, Xin H, Gu J, et al: Solid tumor penetration by integrinmediated pegylated poly(trimethylene carbonate) nanoparticles loaded with paclitaxel. Biomaterials 34: 1739-1746, 2013.

8. Han L, Huang R, Liu S, et al: Peptide-conjugated PAMAM for targeted doxorubicin delivery to transferrin receptor overexpressed tumors. Mol Pharm 7: 2156-2165, 2010.

9. Mei D, Gao H, Gong W, et al: Anti glioma effect of doxorubicin loaded liposomes modified with angiopep-2. Afr J Pharm Pharmacol 5: 409-414, 2011.

10. Gao H, Qian J, Yang Z, et al: Whole-cell SELEX aptamerfunctionalised poly(ethyleneglycol)-poly( $\varepsilon$-eaprolactone nanoparticles for enhanced targeted glioblastoma therapy. Biomaterials 33: 6264-6272, 2012.

11. Zhang L, Chan JM, Gu FX, et al: Self-assembled lipid - polymer hybrid nanoparticles: a robust drug delivery 2: $1696-1702,2008$.

12. Owen SC, Patel N, Logie J, et al: Targeting HER2 breast cancer cells: lysosomal accumulation of anti-HER2 antibodies is influenced by antibody binding site and conjugation to polymeric nanoparticles. J Control Release 172: 395-404, 2013.

13. Rossiello R, Carriero MV and Giordano GG: Distribution of ferritin, transferrin and lactoferrin in breast carcinoma tissue.
J Clin Pathol 37: 51-55, 1984.

14. Shindelman JE, Ortmeyer AE and Sussman HH: Demonstration of the transferrin receptor in human breast cancer tissue. Potential marker for identifying dividing cells. Int J Cancer 27 329-334, 1

15. Han L, Li J, Huang S, et al: Peptide-conjugated polyamidoamine dendrimer as a nanoscale tumor-targeted $\mathrm{T} 1$ magnetic resonance imaging contrast agent. Biomaterials 32: 2989-2998, 2011.

16. Lee JH, Engler JA, Collawn JF and Moore BA: Receptor mediated uptake of peptides that bind the human transferrin receptor. Eur J Biochem 268: 2004-2012, 2001.

17. Oh S, Kim BJ, Singh NP, et al: Synthesis and anti-cancer activity of covalent conjugates of artemisinin and a transferrin-receptor targeting peptide. Cancer Lett 274: 33-39, 2009.

18. Sharma G, Modgil A, Sun C, Singh J, et al: Grafting of cellpenetrating peptide to receptor-targeted liposomes improves their transfection efficiency and transport across blood-brain barrier model. J Pharm Sci 101: 2468-2478, 2012.

19. Kibria G, Hatakeyama H, Ohga N, et al: Dual-ligand modification of PEGylated liposomes shows better cell selectivity and efficient gene delivery. J Control Release 153: 141-148, 2011.
20. Qin Y, Chen H, Zhang Q, et al: Liposome formulated with TAT-modified cholesterol for improving brain delivery and therapeutic efficacy on brain glioma in animals. Int J Pharm 420: 304-312, 2011

21. Gupta B, Levchenko TS and Torchilin VP: Intracellular delivery of large molecules and small particles by cell-penetrating proteins and peptides. Adv Drug Deliv Rev 57: 637-651, 2005.

22. Banks WA, Robinson SM, Nath A, et al: Permeability of the blood-brain barrier to HIV-1 Tat. Exp Neurol 193: 218-227, 2005.

23. Brooks H, Lebleu B and Vivès E: Tat peptide-mediated cellular delivery: back to basics. Adv Drug Deliv Rev 57: 559-577, 2005.

24. Torchilin VP, Rammohan R, Weissig V and Levchenko TS: TAT peptide on the surface of liposomes affords their efficient intracellular delivery even at low temperature and in the presence of metabolic inhibitors. Proc Nat Acad Sci USA 98: 8786-8791, 2001.

25. Allen TM, Brandeis E, Hansen CB, et al: A new strategy for attachment of antibodies to sterically stabilized liposomes resulting in efficient targeting to cancer cells. Biochim Biophys Acta 1237: 99-108, 1995

26. Du S, Pan H, Lu W, et al: Cyclic Arg-Gly-Asp peptide-labeled liposomes for targeting drug therapy of hepatic fibrosis in rats. J Pharmacol Exp

27. Li F, Sun J, Wang J, et al: Effect of hepatocyte growth factor encapsulated in targeted liposomes on Viver cirrhosis. J Control Release 131: 77-82,

28. Gao LY, Liu XY, Chen CJ, et al: Core-shell type lipid/rPAA-Chol polymer hybrid nanoparticles for in vivo siRNA delivery. Biomaterials 35: 2066-2078, 2014.

29. MeNeeley KM, Karathanasis E, Annapragada AV, Bellamkonda RV: Masking and triggered unmasking of argeting ligands on nanocarriers to improve drug delivery to brain tumors. Biomaterials 30: 3986-3995, 2009.

30. Kuai R, Yuan W, Qin Y, et al: Efficient delivery of payload into tumor cells in a controlled manner by TAT and thiolytic cleavable PEG co-modified liposomes. Mol Pharm 7: 18161826,2010

31. Jiang T, Zhang Z, Zhang Y, et al: Dual-functional liposomes based on $\mathrm{pH}$-responsive cell-penetrating peptide and hyaluronic acid for tumor-targeted anticancer drug delivery. Biomaterials 33: 9246-9258, 2012.

Maeda N, Takeuchi Y, Takada M, et al: Anti-neovascular therapy by use of tumor neovasculature-targeted long-circulating liposome. J Control Release 100: 41-52, 2004.

33. Yang T, Choi MK, Cui FD, Kim JS, et al: Preparation and evaluation of paclitaxel-loaded PEGylated immunoliposome. J Control Release 120: 169-177, 2007.

34. Liu Y, Li K, Pan J, et al: Folic acid conjugated nanoparticles of mixed lipid monolayer shell and biodegradable polymer core for targeted delivery of Docetaxel. Biomaterials 31: 330-338, 2010

35. Lewis CE and Pollard JW: Distinct role of macrophages in different tumor microenvironments. Cancer Res 66: 605-612, 2006.

36. Fukumura D, Xu L, Chen Y, Gohongi T, Seed B and Jain RK: Hypoxia and acidosis independently up-regulate vascular endothelial growth factor transcription in brain tumors in vivo. Cancer Res 61: 6020-6024, 2001

37. Jain RK: Delivery of molecular and cellular medicine to solid tumors. Adv Drug Deliv Rev 46: 149-168, 2001.

38. Kobayashi H, Man S, Graham CH, et al: Acquired multicellularmediated resistance to alkylating agents in cancer. Proc Natl Acad Sci USA 90: 3294-3298, 1993.

39. Du J, Lu WL, Ying X, et al: Dual-targeting topotecan liposomes modified with tamoxifen and wheat germ agglutinin significantly improve drug transport across the blood-brain barrier and survival of brain tumor-bearing animals. Mol Pharm 6: 905-917, 2009. 